\title{
Nuclear physics in soft-wall AdS/QCD: Deuteron electromagnetic form factors
}

\author{
Thomas Gutsche, ${ }^{1}$ Valery E. Lyubovitskij,,${ }^{1,2,3}$ Ivan Schmidt,${ }^{4}$ and Alfredo Vega ${ }^{5,6}$ \\ ${ }^{1}$ Institut für Theoretische Physik, Universität Tübingen, \\ Kepler Center for Astro and Particle Physics, Auf der Morgenstelle 14, D-72076 Tübingen, Germany \\ ${ }^{2}$ Department of Physics, Tomsk State University, 634050 Tomsk, Russia \\ ${ }^{3}$ Mathematical Physics Department, Tomsk Polytechnic University, Lenin Avenue 30, 634050 Tomsk, Russia \\ ${ }^{4}$ Departamento de Física y Centro Científico Tecnológico de Valparaíso (CCTVal), \\ Universidad Técnica Federico Santa María, Casilla 110-V, Valparaíso, Chile \\ ${ }^{5}$ Instituto de Física y Astronomía, Universidad de Valparaíso, Avenida Gran Bretaña 1111, Valparaíso, Chile \\ ${ }^{6}$ Centro de Astrofísica de Valparaíso, Avenida Gran Bretaña 1111, Valparaíso, Chile
}

(Dated: April 9, 2018)

\begin{abstract}
We present a high-quality description of the deuteron electromagnetic form factors in a soft-wall AdS/QCD approach. We first propose an effective action describing the dynamics of the deuteron in the presence of an external vector field. Based on this action the deuteron electromagnetic form factors are calculated, displaying the correct $1 / Q^{10}$ power scaling for large $Q^{2}$ values. This finding is consistent with quark counting rules and the earlier observation that this result holds in confining gauge/gravity duals. The $Q^{2}$ dependence of the deuteron form factors is defined by a single and universal scale parameter $\kappa$, which is fixed from data.
\end{abstract}

PACS numbers: 11.10.Kk,11.25.Tq,12.38.Lg,13.40.Gp

Keywords: deuteron, electromagnetic form factors, gauge-gravity duality, AdS/QCD

The experimental and theoretical study of the deuteron is one of the main focuses of hadronic physics during the last decades (for detailed reviews see e.g. Refs. [1]-[4]). Many theoretical approaches have been applied to the problem of the deuteron form factors: perturbative QCD, chiral effective and phenomenological approaches, potential and quark models (see e.g. Refs. [1]-22]). For example, in potential models the nonrelativistic impuls approximation 7, 8] was used. It leads to deuteron form factors factorized in terms of the isoscalar combinations of the nucleon form factors. These approaches are able to describe data up to $0.5 \mathrm{GeV}^{2}$, but deviate from data for higher $Q^{2}$ and are not consistent with quark counting rules. To include relativistic effects different types of relativistic nuclear models have been developed. One possibility is based on taking into account relativistic corrections in a $v / c$ expansion of the nonrelativistic current (leading to so-called two-body interaction current diagrams) [9 11]. Such approaches are limited in their validity of the description of data up to $1-2 \mathrm{GeV}^{2}$. There is a group of models based on relativistic Hamiltonian constraint dynamics, which uses certain phenomenological potentials (Argonne, Nijmegen, etc.) and three forms of quantization procedures (point, instant or front form) [see e.g. Refs. [12, 13]]. Field-theoretical methods formulated in terms of hadronic (mesons, nucleons, $\Delta$-isobars) degrees of freedom are used in a wide range of approaches. These include models based on the solution of a quasipotential 14 or on Bethe-Salpeter [16] equations. These methods also include field theories quantized on the light cone [17, 18], phenomenological Lagrangian approaches 19] and effective field theories treating the long-range dynamics explicitly while parametrizing the short-distance effects by contact interactions [for recent applications to deuteron form factors see e.g. Refs. 20]]. Another class of approaches supposes to treat the deuteron in terms of fundamental degrees of freedom - quarks and gluons: nonrelativistic quark models [21, 22] and perturbative QCD [6]. The analysis of Ref. [6] results in a prediction for the asymptotic largemomentum-transfer behavior of the deuteron form factors and the form of the deuteron distribution amplitude at short distances. Later on in Ref. 23] it was shown that field theories based on gauge/gravity duality, as proposed in Refs. 24], produce the correct power scaling of hadronic form factors at large momentum transfer. This finding is consistent with the quark counting rules.

The main advantage of our approach is that it gives a description of the deuteron electromagnetic (EM) form factors in terms of a single dimensional parameter $\kappa$ with the correct power scaling $1 / Q^{10}$ at large $Q^{2}$ as predicted by perturbative QCD. Our approach is constructed as a holographic dual to perturbative QCD. It gives a good starting point for studying more complicated many-body nuclear systems.

Encouraged by this property we focus on soft-wall anti-de Sitter/quantum chromodynamics (AdS/QCD) [25 28]. It is a version of a bottom-up approach based on the correspondence of string theory in AdS space and conformal field theory (CFT) in physical space-time. The formalism in soft-wall AdS/QCD is based on an effective action involving 
five-dimensional fields propagating in AdS space, which are dual to the deuteron and the electromagnetic field. We apply our formalism to the calculation of the EM form factors of the deuteron. The deuteron itself is simply considered as a proton-neutron bound state.

Note that there are already some applications of AdS/CFT and AdS/QCD to different problems in nuclear physics: baryon matter at finite temperature and baryon number density [29], cold nuclear matter [30], baryon-charge chemical potential [31] $\rho$ meson condensation at finite isospin chemical potential [32], holographic nuclear matter [33], heavy atomic nuclei [34], nuclear matter to strange matter transition [35], self-bound dense objects [36], and mean-field theory for baryon many-body systems [37] (for reviews see e.g. Refs. [38 [41]).

Our approach is based on an effective action, which in terms of the AdS fields $d^{M}(x, z)$ and $V^{M}(x, z)$, is dual to the Fock component contributing to the deuteron with twist $\tau=6$, and the electromagnetic field, respectively, is given by

$$
\begin{aligned}
S & =\int d^{4} x d z e^{-\varphi(z)}\left[-\frac{1}{4} F_{M N}(x, z) F^{M N}(x, z)-D^{M} d_{N}^{\dagger}(x, z) D_{M} d^{N}(x, z)-i c_{2} F^{M N}(x, z) d_{M}^{\dagger}(x, z) d_{N}(x, z)\right. \\
& +\frac{c_{3}}{4 M_{d}^{2}} e^{2 A(z)} \partial^{M} F^{N K}(x, z)\left(i D_{K} d_{M}^{\dagger}(x, z) d_{N}(x, z)-d_{M}^{\dagger}(x, z) i D_{K} d_{N}(x, z)+\text { H.c. }\right) \\
& \left.+d_{M}^{\dagger}(x, z)\left(\mu^{2}+U(z)\right) d^{M}(x, z)\right],
\end{aligned}
$$

where $A(z)=\log (R / z), F^{M N}(x, z)=\partial^{M} V^{N}(x, z)-\partial^{N} V^{M}(x, z)$ is the stress tensor of the vector field $V^{M}(x, z)$, $D^{M}=\partial^{M}-i e V^{M}(x, z)$ is the covariant derivative, $\mu^{2} R^{2}=(\Delta-1)(\Delta-3)$ is the five-dimensional mass; $R$ is the AdS radius, $\varphi(z)=\kappa^{2} z^{2}$ is the background dilaton field; $\Delta=\tau+L$ is the dimension of the $d^{M}(x, z)$ field; $L$ is the orbital angular momentum, and $M_{d}$ is the deuteron mass. $U(z)$ is the confinement potential with

$$
U(z)=\frac{\varphi(z)}{R^{2}} U_{0}
$$

where the constant $U_{0}$ is fixed by the value of the deuteron mass. In the following we work in the axial gauge for both vector fields $d^{z}(x, z)=0$ and $V^{z}(x, z)=0$. In our consideration we have two free parameters: $\kappa$ and $U_{0}$ (the latter only relevant for the description of the deuteron mass). As it will be shown later, the parameters $c_{2}$ and $c_{3}$ are constrained by normalization of the deuteron electromagnetic form factors.

First we perform a Kaluza-Klein (KK) decomposition for the vector AdS field dual to the deuteron

$$
d^{\mu}(x, z)=\exp \left[\frac{\varphi(z)-A(z)}{2}\right] \sum_{n} d_{n}^{\mu}(x) \Phi_{n}(z)
$$

where $d_{n}^{\mu}(x)$ is the tower of the KK fields dual to the deuteron fields with radial quantum number $n$ and twist-dimension $\tau=6$, and $\Phi_{n}(z)$ are their bulk profiles.

Then we derive the Schrödinger-type equation of motion (EOM) for the bulk profile $\Phi_{n}(z)$ with

$$
\left[-\frac{d^{2}}{d z^{2}}+\frac{4(L+4)^{2}-1}{4 z^{2}}+\kappa^{4} z^{2}+\kappa^{2} U_{0}\right] \Phi_{n}(z)=M_{d, n}^{2} \Phi_{n}(z)
$$

The analytical solutions of this EOM read

$$
\begin{aligned}
\Phi_{n}(z) & =\sqrt{\frac{2 n !}{(n+L+4) !}} \kappa^{L+5} z^{L+9 / 2} e^{-\kappa^{2} z^{2} / 2} L_{n}^{L+4}\left(\kappa^{2} z^{2}\right), \\
M_{d, n}^{2} & =4 \kappa^{2}\left[n+\frac{L+5}{2}+\frac{U_{0}}{4}\right],
\end{aligned}
$$

where $L_{n}^{m}(x)$ are the generalized Laguerre polynomials. Restricting to the ground state $(n=0, L=0)$ we get $M_{d}=2 \kappa \sqrt{\frac{5}{2}+\frac{U_{0}}{4}}$. Using the central value of data for the deuteron mass $M_{d}=1.875613 \mathrm{GeV}$ and $\kappa=190 \mathrm{MeV}$ (fitted from data on electromagnetic deuteron form factors), we fix $U_{0}=87.4494$. We can compare this value for the deuteron scale parameter to the analogous one of $\kappa_{N}$ defining the nucleon properties - mass and electromagnetic form factors. In Ref. [28] we fixed the value to $\kappa_{N} \simeq 380 \mathrm{MeV}$, which is 2 times bigger than the deuteron scale parameter $\kappa$. The difference between the nucleon and deuteron scale parameters can be related to the change of size of the hadronic systems - the deuteron as a two-nucleon bound state is 2 times larger than the nucleon. 
In the case of the vector field dual to the electromagnetic field we perform a Fourier transform with respect to the Minkowski coordinate

$$
V_{\mu}(x, z)=\int \frac{d^{4} q}{(2 \pi)^{4}} e^{-i q x} V_{\mu}(q) V(q, z)
$$

where $V(q, z)$ is its bulk profile obeying the following EOM:

$$
\partial_{z}\left(\frac{e^{-\varphi(z)}}{z} \partial_{z} V(q, z)\right)+q^{2} \frac{e^{-\varphi(z)}}{z} V(q, z)=0
$$

Its analytical solution [25] can be written in the form of an integral representation introduced in Ref. [42],

$$
V(Q, z)=\kappa^{2} z^{2} \int_{0}^{1} \frac{d x}{(1-x)^{2}} e^{-\kappa^{2} z^{2} x /(1-x)} x^{a}, \quad a=\frac{Q^{2}}{4 \kappa^{2}}, \quad Q^{2}=-q^{2} .
$$

The gauge-invariant matrix element describing the interaction of the deuteron with the external vector field (dual to the electromagnetic field) reads

$$
\begin{aligned}
M_{\mathrm{inv}}^{\mu}\left(p, p^{\prime}\right) & =-\left(G_{1}\left(Q^{2}\right) \epsilon^{*}\left(p^{\prime}\right) \cdot \epsilon(p)-\frac{G_{3}\left(Q^{2}\right)}{2 M_{d}^{2}} \epsilon^{*}\left(p^{\prime}\right) \cdot q \epsilon(p) \cdot q\right)\left(p+p^{\prime}\right)^{\mu} \\
& -G_{2}\left(Q^{2}\right)\left(\epsilon^{\mu}(p) \epsilon^{*}\left(p^{\prime}\right) \cdot q-\epsilon^{* \mu}\left(p^{\prime}\right) \epsilon(p) \cdot q\right)
\end{aligned}
$$

where $\epsilon\left(\epsilon^{*}\right)$ and $p\left(p^{\prime}\right)$ are the polarization and four-momentum of the initial (final) deuteron, and $q=p^{\prime}-p$ is the momentum transfer. The three EM form factors $G_{1,2,3}$ of the deuteron are related to the charge $G_{C}$, quadrupole $G_{Q}$ and magnetic $G_{M}$ form factors by

$$
G_{C}=G_{1}+\frac{2}{3} \tau_{d} G_{Q}, \quad G_{M}=G_{2}, \quad G_{Q}=G_{1}-G_{2}+\left(1+\tau_{d}\right) G_{3}, \quad \tau_{d}=\frac{Q^{2}}{4 M_{d}^{2}} .
$$

These form factors are normalized at zero recoil as

$$
G_{C}(0)=1, \quad G_{Q}(0)=M_{d}^{2} \mathcal{Q}_{d}=25.83, \quad G_{M}(0)=\frac{M_{d}}{M_{N}} \mu_{d}=1.714,
$$

where $M_{d}$ and $M_{N}$ are deuteron and nucleon masses, and $\mathcal{Q}_{d}=7.3424 \mathrm{GeV}^{-2}$ and $\mu_{d}=0.8574$ are the quadrupole and magnetic moments of the deuteron. Since the deuteron is a spin-1 particle it has three EM form factors in the one-photon-exchange approximation, due to current conservation and the $P$ and $C$ invariance of the EM interaction.

We illustrate the algorithm for calculating the deuteron form factors, considering a particular case of the form factor $G_{1}\left(Q^{2}\right)$, which is generated by the second term in the effective action (1),

$$
S^{(1)}=\int d^{4} x d z e^{-\varphi(z)} e V_{\mu}(x, z)\left(i \partial^{\mu} d_{\nu}^{\dagger}(x, z) d^{\nu}(x, z)-d_{\nu}^{\dagger}(x, z) i \partial^{\mu} d^{\nu}(x, z)\right) .
$$

Next we use the Kaluza-Klein decomposition (3) for the five-dimensional fields $d_{\nu}(x, z)$ and $d_{\nu}^{\dagger}(x, z)$ (restricting to the contribution of the ground states with $n=0)$ and perform the Fourier transform for $d_{\nu}(x), d_{\nu}^{\dagger}(x)$,

$$
d_{\nu}(x)=\int \frac{d^{4} p}{(2 \pi)^{4}} e^{-i p x} \epsilon_{\nu}(p), \quad d_{\nu}^{\dagger}(x)=\int \frac{d^{4} p^{\prime}}{(2 \pi)^{4}} e^{i p^{\prime} x} \epsilon_{\nu}^{*}\left(p^{\prime}\right)
$$

and $V_{\mu}(x, z)$ [see Eq. (6) ]. Substituting expressions (6) and (13) in action (12) and integrating over $x$ and $z$, we get

$$
S^{(1)}=(2 \pi)^{4} \int \frac{d^{4} p}{(2 \pi)^{4}} \int \frac{d^{4} p^{\prime}}{(2 \pi)^{4}} \int \frac{d^{4} q}{(2 \pi)^{4}} \delta^{4}\left(p+q-p^{\prime}\right) e V_{\mu}(q) M_{\mathrm{inv}}^{\mu,(1)}\left(p, p^{\prime}\right)
$$

where $M_{\text {inv }}^{\mu,(1)}\left(p, p^{\prime}\right)$ is part of the invariant matrix element of the $d+\gamma \rightarrow d$ transition containing the contribution of the form factor $G_{1}\left(Q^{2}\right)$

$$
M_{\mathrm{inv}}^{\mu,(1)}\left(p, p^{\prime}\right)=-\left(p+p^{\prime}\right)^{\mu} \epsilon^{*}\left(p^{\prime}\right) \cdot \epsilon(p) G_{1}\left(Q^{2}\right)
$$


In our approach the deuteron form factor $G_{1}\left(Q^{2}\right)=F\left(Q^{2}\right)$, where $F\left(Q^{2}\right)$ is the twist-6 hadronic form factor, which is given by the overlap of the square of the bulk profile dual to the deuteron wave function (twist- 6 hadronic wave function) and the confined electromagnetic current

$$
F\left(Q^{2}\right)=\int_{0}^{\infty} d z \Phi_{0}^{2}(z) V(Q, z)=\frac{\Gamma(6) \Gamma(a+1)}{\Gamma(a+6)}
$$

where $a=Q^{2} /\left(4 \kappa^{2}\right)$. This formula follows from the general and universal formula for the hadronic form factor with twist $\tau$ derived in Ref. [27] in terms of the bulk profile $\phi_{\tau}(z)=\sqrt{\frac{2}{(\tau-2) !}} \kappa^{\tau-1} z^{\tau-3 / 2} e^{-\kappa^{2} z^{2} / 2}$ dual to the hadronic wave function with twist $\tau$ :

$$
F_{\tau}\left(Q^{2}\right)=\int_{0}^{\infty} d z \phi_{\tau}^{2}(z) V(Q, z)=\frac{\Gamma(\tau) \Gamma(a+1)}{\Gamma(a+\tau)}
$$

Therefore, Eq. (16) is the particular case of Eq. (17) for $\tau=6$.

By analogy we calculate the other two deuteron form factors $G_{2}$ and $G_{3}$, which are expressed in terms of the same universal factor $F\left(Q^{2}\right)$ :

$$
G_{i}\left(Q^{2}\right)=c_{i} F\left(Q^{2}\right), \quad i=2,3
$$

The parameters $c_{2}$ and $c_{3}$ are defined by normalization of the deuteron form factors as:

$$
c_{2}=G_{M}(0)=1.714, \quad c_{3}=G_{M}(0)+G_{Q}(0)-1=26.544 .
$$

Note that the form factor $F\left(Q^{2}\right)$ has the correct power-scaling $F\left(Q^{2}\right) \sim 1 /\left(Q^{2}\right)^{5}$ at large $Q^{2} \rightarrow \infty$. It can also be written in the Brodsky-Ji-Lepage form derived within perturbative QCD. The deuteron form factor is factorized in terms of the nucleon form factor $F_{N}\left(Q^{2} / 4\right)$ and the so-called "reduced" nuclear form factor $f_{d}\left(Q^{2}\right)[6]$ : $F_{d}\left(Q^{2}\right)=$ $f_{d}\left(Q^{2}\right) F_{N}^{2}\left(Q^{2} / 4\right)$. Our result reads

$$
F_{d}\left(Q^{2}\right) \equiv F\left(Q^{2}\right)=\frac{\Gamma(6) \Gamma(a+1)}{\Gamma(a+6)}=\frac{5 !}{(a+1) \ldots(a+5)}=f_{d}\left(Q^{2}\right) F_{N}^{2}\left(Q^{2} / 4\right)
$$

where our predictions for $f_{d}\left(Q^{2}\right)$ and $F_{N}\left(Q^{2} / 4\right)$ are

$$
f_{d}\left(Q^{2}\right)=\frac{30(a+1)(a+2)}{(a+3)(a+4)(a+5)}, \quad F_{N}\left(Q^{2} / 4\right)=\frac{2}{(a+1)(a+2)}
$$

where $a=Q^{2} /\left(4 \kappa^{2}\right)$. Our results for the charge $G_{C}\left(Q^{2}\right)$, quadrupole $G_{Q}\left(Q^{2}\right)$ and magnetic $G_{M}\left(Q^{2}\right)$ form factors are shown in Figs.1-3. The shaded band corresponds to values of the scale parameter $\kappa$ in the range of $150 \mathrm{MeV}<\kappa<$ $250 \mathrm{MeV}$. An increase of the parameter $\kappa$ leads to an enhancement of the form factors. The best description of the data on the deuteron form factors is obtained for $\kappa=190 \mathrm{MeV}$ and is shown by the solid line. Data points are taken from Refs. [2, 4]. To quantify the quality of the fit with $\kappa=190 \mathrm{MeV}$ we indicate the $\chi^{2}$ values for the three deuteron form factors: $\chi^{2}=0.2$ for $\left(G_{C}\right), \chi^{2}=13.8$ for $\left(G_{Q}\right)$ and $\chi^{2}=2.3$ for $\left(G_{M}\right)$. We would also like to point out that with $\kappa=190 \mathrm{MeV}$ our result for the deuteron charge radius $r_{C}=\left(-6 d G_{C}\left(Q^{2}\right) /\left.d Q^{2}\right|_{Q^{2}=0}\right)^{1 / 2}=\sqrt{\frac{137}{40 \kappa^{2}}-\mathcal{Q}_{d}}=1.846$ fm compares well with data, $r_{C}=2.130 \pm 0.010 \mathrm{fm}[1]$.

In conclusion we stress again the main result of this paper. Using the soft-wall AdS/QCD model we calculate the deuteron electromagnetic form factors, which are given by analytical expressions in terms of a universal twist- 6 form factor $F\left(Q^{2}\right)$ relevant for the deuteron — hadronic system with six partons. Our framework gives a description of the deuteron in terms of two free parameters - the dimensional parameter $\kappa$ and the confinement parameter $U_{0}$. The parameter $\kappa$ is fixed by the scale of the deuteron form factors and the parameter $U_{0}$ is fixed through the deuteron mass.

This work was supported by the German Bundesministerium für Bildung und Forschung (BMBF) under Grant No. 05P12VTCTG, by Marie Curie Reintegration Grant IRG 256574, by CONICYT (Chile) Research Project No. 80140097, by CONICYT (Chile) under Grant No. 7912010025, by FONDECYT (Chile) under Grants No. 1140390 and No. 1141280, and by Tomsk State University Competitiveness Improvement Program. V.E.L. would like to thank 
Departamento de Física y Centro Científico Tecnológico de Valparaíso (CCTVal), Universidad Técnica Federico Santa María, Valparaíso, Chile and Instituto de Física y Astronomía, Centro de Astrofísica de Valparaíso, Universidad de Valparaíso, Chile for warm hospitality.

[1] M. Garcon and J. W. Van Orden, Adv. Nucl. Phys. 26, 293 (2001).

[2] D. Abbott et al. (JLAB t20 Collaboration), Eur. Phys. J. A 7, 421 (2000).

[3] M. Kohl, Nucl. Phys. A 805, 361 (2008).

[4] R. J. Holt and R. Gilman, Rep. Prog. Phys. 75, 086301 (2012).

[5] R. G. Arnold, C. E. Carlson, and F. Gross, Phys. Rev. C 21, 1426 (1980); Phys. Rev. C 23, 363 (1981).

[6] S. J. Brodsky, C. R. Ji, and G. P. Lepage, Phys. Rev. Lett. 51, 83 (1983).

[7] T. De Forest, Jr. and J. D. Walecka, Adv. Phys. 15, 1 (1966).

[8] T. W. Donnelly and J. D. Walecka, Ann. Rev. Nucl. Part. Sci. 25, 329 (1975).

[9] J. L. Friar, Phys. Rev. C 12, 695 (1975).

[10] J. F. Mathiot, Phys. Rept. 173, 63 (1989).

[11] R. Schiavilla and D. O. Riska, Phys. Rev. C 43, 437 (1991).

[12] F. M. Lev, E. Pace, and G. Salme, Phys. Rev. Lett. 83, 5250 (1999).

[13] T. W. Allen, G. L. Payne, and W. N. Polyzou, Phys. Rev. C 62, 054002 (2000).

[14] R. Blankenbecler and R. Sugar, Phys. Rev. 142, 1051 (1966).

[15] A. A. Logunov and A. N. Tavkhelidze, Nuovo Cim. 29, 380 (1963).

[16] W. W. Buck and F. Gross, Phys. Rev. D 20, 2361 (1979)

[17] J. Carbonell, B. Desplanques, V. A. Karmanov, and J. F. Mathiot, Phys. Rep. 300, 215 (1998).

[18] V. A. Karmanov and A. V. Smirnov, Nucl. Phys. A 546, 691 (1992).

[19] Y. B. Dong, A. Faessler, T. Gutsche, and V. E. Lyubovitskij, Phys. Rev. C 78, 035205 (2008).

[20] S. Kolling, E. Epelbaum, and D. R. Phillips, Phys. Rev. C 86, 047001; E. Epelbaum, A. M. Gasparyan, J. Gegelia, and M. R. Schindler, Eur. Phys. J. A 50, 51 (2014).

[21] A. Buchmann, Y. Yamauchi, and A. Faessler, Phys. Lett. B 225, 301 (1989).

[22] H. Ito and L. S. Kisslinger, Phys. Rev. C 40, 887 (1989).

[23] J. Polchinski and M. J. Strassler, Phys. Rev. Lett. 88, 031601 (2002).

[24] J. M. Maldacena, Adv. Theor. Math. Phys. 2, 231 (1998) [Int. J. Theor. Phys. 38, 1113 (1999)]; S. S. Gubser, I. R. Klebanov, and A. M. Polyakov, Phys. Lett. B 428, 105 (1998); E. Witten, Adv. Theor. Math. Phys. 2, 253 (1998).

[25] A. Karch, E. Katz, D. T. Son, and M. A. Stephanov, Phys. Rev. D 74, 015005 (2006).

[26] O. Andreev, Phys. Rev. D 73, 107901 (2006).

[27] S. J. Brodsky and G. F. de Teramond, Phys. Rev. D 77, 056007 (2008).

[28] T. Gutsche, V. E. Lyubovitskij, I. Schmidt, and A. Vega, Phys. Rev. D 85, 076003 (2012); Phys. Rev. D 86, 036007 (2012); Phys. Rev. D 87, 016017 (2013); Phys. Rev. D 87, 056001 (2013); A. Vega, I. Schmidt, T. Branz, T. Gutsche, and V. E. Lyubovitskij, Phys. Rev. D 80, 055014 (2009); T. Branz, T. Gutsche, V. E. Lyubovitskij, I. Schmidt, and A. Vega, Phys. Rev. D 82, 074022 (2010).

[29] O. Bergman, G. Lifschytz, and M. Lippert, JHEP 11, 056 (2007).

[30] M. Rozali, H. H. Shieh, M. Van Raamsdonk, and J. Wu, JHEP 01, 053 (2008).

[31] S. Nakamura, Y. Seo, S. J. Sin and K. P. Yogendran, Prog. Theor. Phys. 120, 51 (2008).

[32] O. Aharony, K. Peeters, J. Sonnenschein, and M. Zamaklar, JHEP 02, 071 (2008).

[33] Y. Kim, C. H. Lee, and H. U. Yee, Phys. Rev. D 77, 085030 (2008).

[34] K. Hashimoto, Prog. Theor. Phys. 121, 241 (2009).

[35] Y. Kim, Y. Seo, and S. J. Sin, JHEP 03, 074 (2010).

[36] K. K. Kim, Y. Kim, and Y. Ko, JHEP 10, 039 (2010).

[37] M. Harada, S. Nakamura, and S. Takemoto, Phys. Rev. D 86, 021901 (2012).

[38] Y. Kim and D. Yi, Adv. High Energy Phys. 2011, 259025 (2011).

[39] S. Aoki, K. Hashimoto, and N. Iizuka, Rep. Prog. Phys. 76, 104301 (2013).

[40] Y. Kim, I. J. Shin, and T. Tsukioka, Prog. Part. Nucl. Phys. 68, 55 (2013).

[41] M. R. Pahlavani and R. Morad, Adv. High Energy Phys. 2014, 863268 (2014).

[42] H. R. Grigoryan and A. V. Radyushkin, Phys. Rev. D 76, 095007 (2007). 


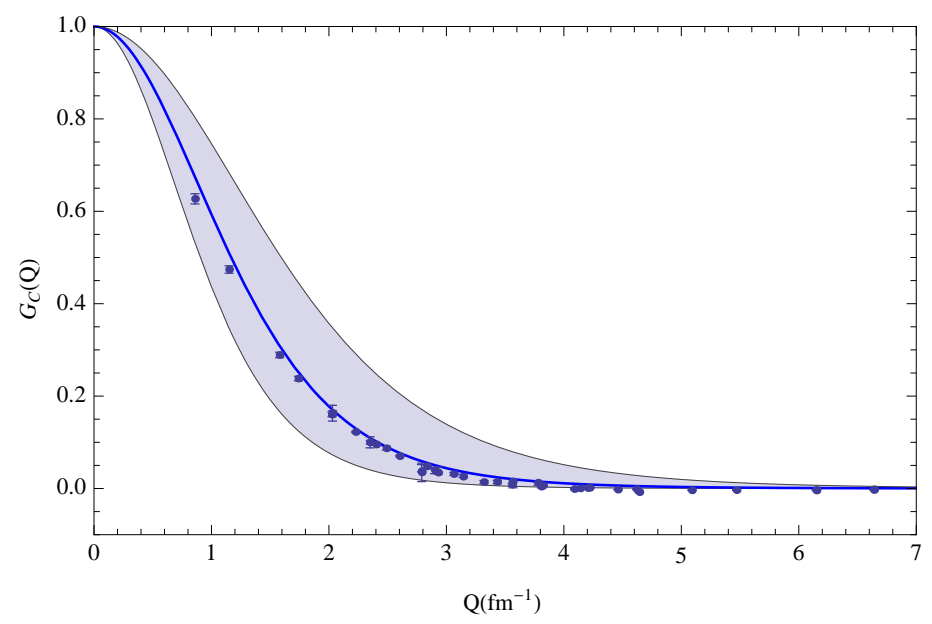

FIG. 1: Charge deuteron form factor.

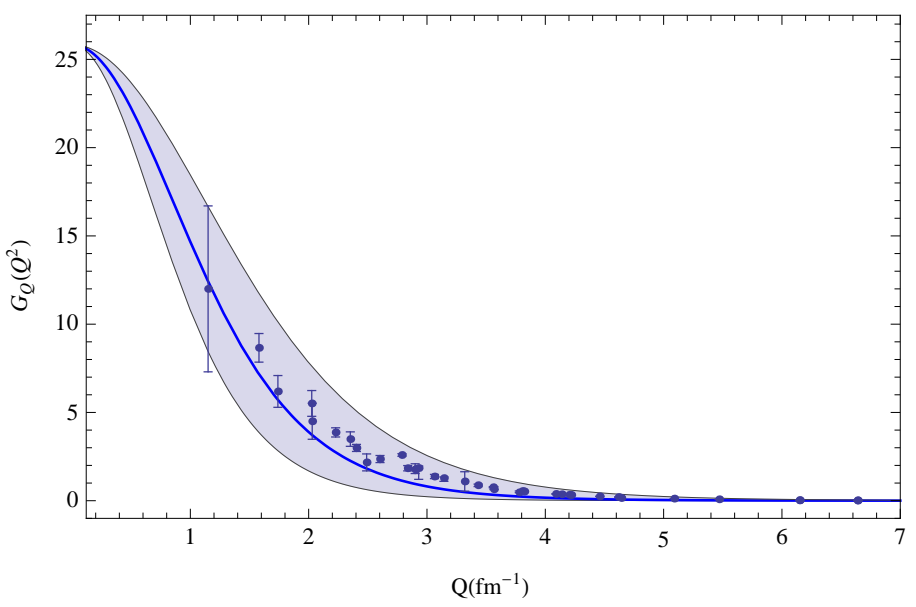

FIG. 2: Quadrupole deuteron form factor.

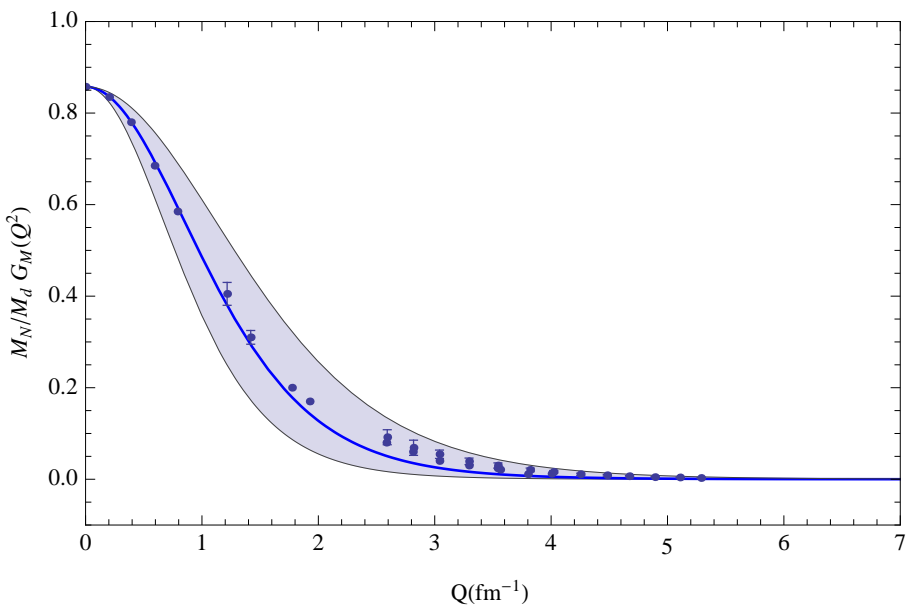

FIG. 3: Magnetic deuteron form factor. 